\title{
Benign form of multiple sclerosis: MRI evidence for less frequent and less inflammatory disease activity
}

\author{
D Kidd, A J Thompson, B E Kendall, D H Miller, W I McDonald
}

\begin{abstract}
Data from two serial studies comparing the MRI activity of two groups of 11 patients, one with early relapsing-remitting (mean disease duration $3 \cdot 5$ years), the other benign (mean disease duration 22 years) multiple sclerosis are presented. Those with benign disease developed fewer new or enlarging lesions, and such lesions that occurred had a lower incidence of gadolinium enhancement, a marker of inflammation. These results, when considered in conjunction with other published studies, suggest that both the frequency of new lesions and the amount of inflammation within them influence the development of disability in multiple sclerosis.
\end{abstract}

$(\mathcal{F}$ Neurol Neurosurg Psychiatry 1994;57:1070-1072)

The benign form of multiple sclerosis is defined as the presence of minimal disability at least 10 years after the onset of symptoms ${ }^{1}$; its incidence has been cited as between 15 and $40 \%{ }^{2-5}$ depending on the criteria used. The pathophysiological mechanisms that lead to a benign course in some patients, and the accumulation of severe disability in others, are poorly understood. It might be expected that MRI would provide new insights into these mechanisms, as it shows much of the ongoing pathological process in vivo. ${ }^{6}$ Cross sectional MRI studies, however, have shown little or no relation between clinical disability and MRI lesion load in either the brain ${ }^{7}$ or the spinal cord, ${ }^{8}$ which suggests that the extent of lesions visible on conventional MRI does not provide a sufficient explanation for differing clinical courses.

Longitudinal MRI studies, which document the evolution of lesions over time, provide additional information about disease activity. In a previous study we reported two small groups of relapsing-remitting patients with multiple sclerosis with different disease durations-early relapsing-remitting and benign multiple sclerosis-who underwent monthly brain MRI for six months. ${ }^{9}$ During this period, the benign group developed fewer new lesions, and a smaller proportion of the new lesions displayed gadolinium enhancement. Because of the small numbers of patients, this was not a definitive study. We therefore enlarged the earlier cohorts of patients with early relapsing-remitting and benign multiple sclerosis with the same study design, and now present the combined results of the two studies, with particular emphasis on the insights they provide into the mechanisms of disability in multiple sclerosis.

\section{Patients and methods}

Twelve patients with clinically definite multiple sclerosis ${ }^{10}$ were selected from the patient population attending the National Hospitals for Neurology and Neurosurgery. All had oligoclonal bands in the CSF and all gave informed written consent to enter the study. Selection was based on willingness to participate and on disability state (expanded disability status scale (EDSS) $\leqslant 3 \cdot 0$ ). Six patients had an early relapsing-remitting course defined as a disease duration of less than five years, six had a benign course defined as disease duration of greater than 10 years.

Patients underwent a full neurological examination at the onset of the study and MRI of the brain with gadolinium-DTPA enhancement. They were followed up with gadolinium enhanced MRI at monthly intervals for six months. At each visit they were questioned about new or recurring symptoms and were re-examined.

\section{MRI PROTOCOL}

Brain MR scans were obtained on a Signa 1.5T superconducting system (GE Milwaukee). T2 weighted and proton density weighted images were acquired with $345 \mathrm{~mm}$ contiguous axial slices parallel to a plane between the anterior and posterior limbs of the corpus callosum using a variable spin echo sequence $\mathrm{SE}_{2500 / 32,80}$. Five minutes after injection of $0.1 \mathrm{mmol} / \mathrm{kg}$ gadolinium-DTPA (Magnevist) a $\mathrm{T} 1$ weighted sequence $\mathrm{SE}_{500,19}$ was acquired. Images were plotted in a $256 \times 192$ image matrix with a $24 \mathrm{~cm}$ field of view. The scans were examined by a neuroradiologist (BK) who was blinded to the clinical information. Lesions were counted in five periventricular and eight non-periventricular white matter regions, and lesions were graded according to their longest diameter as $<5 \mathrm{~mm}$, 5-10 mm, >10 mm, or confluent. Lesions were scored as being 1-4 respectively depending on this measurement. Total brain lesion load was calculated by adding the lesion size scores on the first scan. The occurrence of new and enlarging lesions with or without enhancement was noted over time. The study was approved by the joint medical ethics committee of the National Hospital and Institute of Neurology. 
Clinical and MRI characteristics of the present study and combined studies

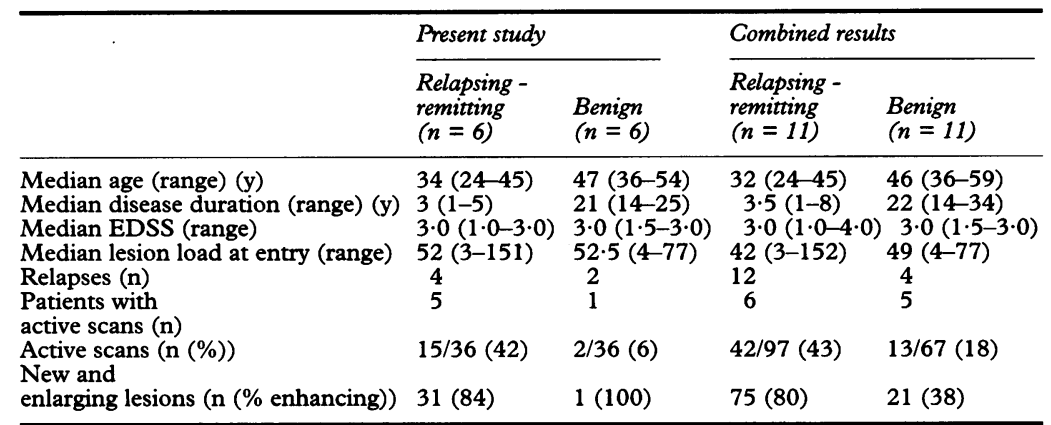

The data for the 12 patients in the present study are presented separately and in combination with that obtained from the 10 patients previously studied by a similar protocol at $0.5 \mathrm{~T}$ field strength (table). No patient in either clinical group participated in both studies.

\section{STATISTICAL METHODS}

Groups were compared with $\chi^{2}$ and MannWhitney $U$ tests for parametric and non-parametric data respectively.

\section{Results}

\section{PRESENT STUDY}

There was no significant difference in mean lesion count or load of the first scans between the two groups. Over the six month study period there was a total of four clinical relapses in the early relaping-remitting group and two in the benign group. Four patients in each group were clinically unchanged (table). There were 31 new or enlarging lesions in the early relapsing-remitting group of which 26 enhanced and in the benign group there was one new lesion, which also enhanced. Of the four patients in the early relapsing-remitting group who had had no clinical activity the scans of one patient were unchanged over the study period and one new lesion occurred in each of the other three, one of which was seen to enhance. In the other two patients two relapses occurred in each; they were associated with 17 new or enlarging lesions in one case (14 of which enhanced), and 11 in the other (all of which enhanced).

In the benign group the single new lesion enhanced and occurred without clinical symptoms. The two patients who relapsed did so without cerebral MRI change. One relapse was due to a lesion located on clinical grounds in the pons, the other in the spinal cord. The number of new and enlarging lesions differed significantly between the groups $(p<0.03)$.

\section{COMBINED STUDIES}

The table shows the amalgamated data from the present and the previous studies. Whereas there was little difference in the number of patients showing MRI activity at any point in the study in the two groups, there was a clear difference in the extent of MRI activity both in terms of the percentage of active scans $(p=0.00012)$ and the number of active lesions $(p=0.03)$. There was a significant difference in the number of new or enlarging lesions per patient per relapse between the two groups $(6.25$ in the early relapsingremitting group $v 2.25$ in the benign group, $\mathrm{p}<0.03)$, in the number of new or enlarging lesions per patient (6.8 in the early relapsingremitting group $v 1.9$ in the benign group, $p<0.03$ ), and in the proportion of those that displayed enhancement $(80 \%$ in the early relapsing-remitting group $v 38 \%$ in the benign group, $\mathrm{p}<0.0001)$. There was no relation between the age of the patient and the frequency of active lesions on MRI ( $p>0 \cdot 1)$.

\section{Discussion}

The present study confirms our earlier findings. Combining the results of the two studies gives a cohort of 11 patients each with early relapsing-remitting and benign multiple sclerosis, and the results show that active lesions appear about one third as often in those with benign multiple sclerosis. Furthermore, new or enlarging lesions showed gadoliniumDTPA enhancement significantly less often in benign multiple sclerosis. As gadolinium enhancement is a marker of blood-brain barrier breakdown, which in multiple sclerosis correlates with the presence of inflammation, ${ }^{11}$ the decreased frequency of enhancement suggests a less severe or shorter inflammatory phase in the development of new lesions in benign multiple sclerosis.

An important question, not answered by the present study, is whether the differences in disease activity are a consequence of differences in the duration of the disease rather than in its severity. Clinical relapses become less frequent with increasing disease duration, ${ }^{1}$ so it is plausible that the number of active MRI lesions will also diminish. This question could be considered by studying a group of disabled relapsing-remitting or secondary progressive patients matched with the benign group for disease duration (patients with primary progressive multiple sclerosis and without superimposed relapses should not be included as they have a much smaller rate of MRI activity than secondary progressive patients matched for disease duration and disability). ${ }^{12}$ A second issue is the possible effect of age on MRI activity; although no significant relation was found in this study, a possible reduction in MRI activity with advancing age cannot be excluded.

Although data are not available for disabled relapsing-remitting or secondary progressive patients with the same disease duration as our benign group, two studies of cohorts with a shorter disease duration suggest that the amount of MRI activity will be predictive of the long term clinical course. Firstly, ${ }^{12}$ a group of 12 disabled patients with secondary progressive multiple sclerosis, with a mean disease duration $(7 \cdot 8$ years) intermediate between the present early relapsing-remitting and benign groups, displayed an amount of MRI activity (mean $18 \cdot 2$ lesions per patient per year) and enhancement ( $87 \%$ of lesions) 
similar to that seen in the early relapsingremitting group and much in excess of that seen in the benign group.

Secondly, a recent five year follow up study of patients first scanned at presentation with a single episode compatible with demyelination (for example, optic neuritis), showed a strong correlation between the number of brain MRI lesions at presentation and the number of new lesions as well as the degree of clinical disability at follow up. ${ }^{13}$ Usually patients without disability had developed few new lesions during the preceding five years, and it is likely that many of these will go on to have benign disease, as it has been shown that the absence of appreciable disability after five years of clinical symptoms is associated with a high probability of remaining minimally disabled over the next $10-15$ years. ${ }^{14} 15$

Thus studies to date in multiple sclerosis of both short and long disease duration, suggest that a low frequency of new lesion development is usually predictive of a benign course. This is not surprising. It is self evident that a low rate of new lesion development means a smaller chance that there will be involvement of a clinically vulnerable pathway liable to cause disability-for example, the corticospinal tract. Notwithstanding, it must be emphasised that the rate of new lesion development is not an absolute predictor of outcome-some patients with a low rate of lesion accrual become disabled, and some with high rates do not. The factors that lead to the development of functional disability are poorly understood, and have been recently reviewed. ${ }^{16}$ They probably include failure of conduction in persistently demyelinated axons, failure of remyelination, and axonal loss. The relative contribution of each is uncertain although given that the capacity for recovery from remyelination is greater than after axonal loss, axonal loss is likely to be important.

How might a less inflammatory disease, as indicated by the low rate of gadoliniumDTPA enhancement, permit a benign clinical course? One possibility is that less severe and less prolonged inflammation results in less extensive demyelination, and in turn a greater capacity for axonal repair (probably by the insertion of sodium channels into the demyelinated axons ${ }^{17}{ }^{18}$ ), and for remyelination after the inflammation has subsided. The second possibility is consistent with the experimental finding that remyelination is slowed by prolonged demyelination, ${ }^{19}$ and by a second episode soon after the first. ${ }^{20}$ It is also possible that prolonged or repeated inflammation at the same site ${ }^{21}$ or different levels of the same fibres might predispose toward axonal disruption. Serial MRI with detailed clinical and electrophysiological correlation should help to elucidate these problems.

The NMR Research Group is funded by a generous grant from the Multiple Sclerosis Society of Great Britain and Northern Ireland and receives additional support from the Medical Research Council. Dr Kidd is funded by the Brain Research Trust. We are grateful to DG MacManus and AJ Brennan for expert radiographic help.

1 Matthews B. Course and prognosis. In: Matthews WB, Allen IV, Compston A, Martyn CN, eds. McAlpine's multiple sclerosis. London: Churchill Livingstone 1991: multiple

2 McAlpine $\mathrm{D}$. The benign form of multiple sclerosis. A study based on 241 cases seen within three years of onset and followed up until the tenth year or more of the disease. Brain 1961;84:186-203.

3 Bonduelle M, Bouygues P, Degos CF, Gauthier C. Les formes bénignes de la Sclerose en Plaques. Rev Neurol 1979;135:593-604.

4 Confavreux C, Aimard G, Devic M. Course and prognosis of multiple sclerosis assessed by the computerised data processing of 349 patients. Brain 1980;103:281-300.

5 Thompson AJ, Hutchinson M, Brazil J, Feighery C, Martin EA. A clinical and laboratory study of benign Martin EA. A clinical and laboratory study
multiple sclerosis. $Q \mathcal{M}$ Med 1986;58:69-80.

6 McDonald WI, Miller DH, Barnes D. The pathological evolution of multiple sclerosis. Neuropathol Appl Neurobiol 1992;18:319-34.

7 Thompson AJ, Kermode AG, MacManus DG, et al. Patterns of disease activity in multiple sclerosis: a clinical and magnetic resonance imaging study. $B M \mathcal{F}$ 1990;300: $631-4$.

8 Kidd D, Thorpe JW, Thompson AJ, et al. Spinal cord maging using multiarray coils and fast spin echo: findings in multiple sclerosis. Neurology 1993;43:2632-7.

9 Thompson AJ, Miller D, Youl B, et al. Serial gadoliniumenhanced MRI in relapsing/remitting multiple sclerosis of varying disease duration. Neurology 1992;42:60-3.

10 Poser CM, Paty DW, Scheinberg L, et al. New diagnostic criteria for multiple sclerosis: guidelines for research protocols. Ann Neurol 1983;13:227-31.

11 Katz D, Taubenberger JK, Cannella B, McFarlin DE, Raine CS, McFarland HF. Correlation between MRI Raine CS, McFarland HF. Correlation between MRI

12 Thompson AJ, Kermode AG, Wicks D, et al. Major differences in the dynamics of primary and secondary progressive multiple sclerosis. Ann Neurol 1991;29: 54-62.

13 Morrisey SP, Miller DH, Kendall BE, et al. The significance of brain magnetic resonance imaging abnormalities at presentation with clinically isolated syndromes suggestive of multiple sclerosis. A five year follow up study. Brain 1993;116:135-46.

14 Miller DH, Hornabrook RW, Purdie G. The natural history of multiple sclerosis: a regional study with some longitudinal data. $\mathcal{F}$ Neurol Neurosurg Psychiatry 1992;55: 341-6.

15 Kurtzke JF, Beebe GW, Nagler B, Kurland LT, Auth Th L. Studies on the natural history of multiple sclerosis. 8 . Studies on the natural history of multiple sclerosis. 8.
Early prognostic features of the later course of the Early prognostic features of the late

16 McDonald WI. Overview of clinical aspects of multiple sclerosis. In: Waxman SG, ed. The axon. 1994 (in press). 17 Waxman SG, Ritchie JM. Organisation of ion channels in the myelinated nerve fiber. Science 1985;228:1502-7.

18 England JD, Gamoni F, Levinson SR, Finger TE. Changed distribution of sodium channels along demyelinated axons. Proc Natl Acad Sci USA 1990;87:6777-80.

19 Ludwin SK. Chronic demyelination inhibits remyelination in the central nervous system. An analysis of contributory factors. Lab Invest 1980;43:382-7.

20 Johnson ES, Ludwin SK. The demonstration of recurrent demyelination and remyelination of axons in the central nervous system. Acta Neuropathol 1981;53:93-8.

21 Prineas JW, Barnard RO, Revesz T, Kwon EE, Sharer L, Cho ES. Multiple sclerosis: pathology of recurrent lesions. Brain 1993;116:681-93. 